\title{
Alineación de la evaluación del desempeño individual a la gestión hotelera
}

\section{Alignment of the evaluation of individual performance to hotel management.}

DrC.. Caridad Leyva Del Toro. ${ }^{1}$

\section{Resumen.}

La evaluación del desempeño individual en las organizaciones turísticas debe responder al logro de los objetivos estratégicos con el acercamiento del trabajador a las expectativas de la dirección; razón que la obliga a ser oportuna, flexible y dinámica. Este trabajo tiene como problema resolver la baja alineación existente entre los resultados individuales con los organizacionales en las entidades hoteleras cubanas, por lo que el objetivo de la investigación es mostrar una tecnología y sus aplicaciones para realizar la evaluación del desempeño individual alineada a la evaluación del desempeño organizacional, con una visión integradora, bajo el uso de tendencias reconocidas internacionalmente en este tema como la gestión por competencias, la mejora continua y el autoperfeccionamiento. La metodología utilizada posee un modelo y procedimiento a partir de la tenencia del cuadro de mando integral operativizado en los niveles estratégico, táctico y operativo, lo que se integra a los indicadores del cargo, los procesos y la entidad. A su vez, se conecta el desempeño individual y el desempeño organizacional a través de conductas estratégicas en las competencias laborales (contempladas para los perfiles de cargos) y los objetivos estratégicos organizacionales.

Palabras claves: evaluación del desempeño individual, alineación, procesos, organización.

\section{Abstract.}

The evaluation of the individual performance in the tourist organizations must respond to the achievement of the strategic objectives with the approach of the worker to the

\footnotetext{
${ }^{1}$ Universidad de Holguín. Cuba. cary@ uho.edu.cu
} 
expectations of the direction; reason that forces it to be timely, flexible and dynamic. This work has as problem to solve the low alignment existing between the individual results with the organizational ones in the Cuban hotel entities, reason why the objective of the investigation is to show a technology and its applications to realize the evaluation of the individual performance aligned to the evaluation of the organizational performance, with an integrating vision, under the use of internationally recognized trends in this subject such as competency management, continuous improvement and self-improvement. The methodology used has a model and procedure based on the possession of the balanced scorecard operationalized at the strategic, tactical and operational levels, which is integrated into the indicators of the position, the processes and the entity. In turn, individual performance and organizational performance are connected through strategic behavior in labor competencies (contemplated for job profiles) and strategic organizational objectives.

Keywords: evaluation of individual performance, alignment, processes, organization.

\section{Introducción.}

El turismo en Cuba ha incidido de forma notable en los cambios tecnológicos, económicos, culturales y sociales, lo que constituye en las últimas décadas, una importante alternativa de desarrollo (Alphonse Browne2, 2014). Hoy se considera el sector más dinámico de la economía cubana (Orellana Alvarado, 2016)3.

El comienzo de siglo XXI tuvo un comportamiento favorable para el aumento progresivo del arribo de turistas al archipiélago cubano. En los últimos 4 años la cifra ha sido de un $5,3 \%$; destacándose el polo holguinero, que en igual periodo muestra un crecimiento promedio del 3\% y su ingreso anual de un 2\%. Se estima desde el 2015 al 2025 un aumento gradual de la planta habitacional con 19000 habitaciones, destacándose Gibara, Centro Ciudad y el Ramón de Antilla (Mintur, 2016).

Las modificaciones administrativas y legales, las exigencias de las partes interesadas, el fenómeno creciente de optimización de las plantillas, el aumento de la demanda de mano de obra más calificada y motivada, son algunos de los factores que han contribuido a que la GCH de las empresas sea más compleja e importante para las mismas. Es por ello que esta actividad debe garantizar el incremento desus indicadores de impacto fundamentales expresados en término del desempeño humano.

En el ámbito de la GCH la evaluación del desempeño individual (EDI), se ha convertido en desafío empresarial en los últimos años, por la búsqueda de su ajuste o correspondencia con la evaluación del desempeño estratégico. Esto se evidencia en las encuestas mundiales realizadas conjuntamente por la Boston ConsultingGroup (BCG) y la

\footnotetext{
${ }^{2}$ Intervención de Gaston Alphonse Browne, primer ministro de Antigua y Barbuda, presidente en ejercicio de CARICOM, en la clausura de la V Cumbre CARICOM-Cuba, La Habana, 8 de diciembre del 2014.

${ }^{3}$ Intervención de María Del Carmen Orellana Alvarado, Directora Comercial del MINTUR, en la mesa redonda del 2 de febrero del 2016.
} 
WorldFederation of Personnel Management Associations (Wfpma), que comprenden a países y empresas de todos los continentes (BCG \&Wfpma, 2008, 2010, 2012).

En una de esas encuestas, se expresó: "Si contemplamos la estrategia, los indicadores de gestión y los RRHH (recursos humanos) como los tres vértices de un triángulo, veremos que en la mayoría de las empresas los vínculos entre RRHH y estrategia así como entre RRHH y los indicadores de gestión están rotos o no existen" 4 . Además se destacó la importancia actual de la EDI y la necesidad de una estrategia sobre esa evaluación. También señalaron que el enfoque de la empresa en la gestión del desempeño, los indicadores de capital humano y los sistemas de incentivos, deberán apoyar con énfasis la consecución de los objetivos estratégicos de la empresa. Aspectos de los cuales Cuba no está ajena.

Estudios desarrollados5 en el campo de la teoría y de la práctica en las empresas demodo general y del sector turístico en particular, han evidenciado que el proceso de EDI se caracteriza por una baja alineación a los restantes procesos de la gestión en general y de los propios de la GCH; insuficiente orientación a la gestión por competencias, limitada capacidad de autoperfeccionamiento y baja correspondencia a los niveles de evaluación del desempeño.

En la actualidad cuando el sector turístico tiene un elevado protagonismo en el desarrollo económico y social del país los sistemas gerenciales deben orientarse hacia los enfoques de dirección más modernos. Esta transformación, tanto de concepción como de métodos que deben utilizarse, esencialmente en lo vinculado a la evaluación del desempeño, deben permitir detectar las reservas de mejora y trazar acciones que respondan a las exigencias estratégicas de las organizaciones y su entorno.

\section{Desarrollo.}

Se desarrolla un procedimiento general para la evaluación del desempeño individual basado en competencias. El mismo cuenta con tres fases (entrada, transformación y salida), seis etapas, 20 pasos y 13 tareas. Se aplicó en el Hotel Brisas Guardalavaca, obteniendo los siguientes resultados:

\section{Fase I. Entrada}

Se crearon las condiciones organizativas para el desarrollo de la evaluación del desempeño individual a través de las dos etapas que la componen y permitieron el conocimiento de elementos estratégicos necesarios en el desempeño organizacional con el personal adecuado para realizarlo.

Se utilizó como fortaleza que la entidad trabajaba en los procedimientos del SGICH, con un trabajo enfocado a los procesos de: selección de personal, comunicación institucional,

${ }^{4}$ Citado por BCG \& WFPMA, 2008, p. 2.

${ }^{5}$ Varela Izquierdo, 2001; Artola Pimentel, 2002; Sotolongo Sánchez, 2005; De Miguel Guzmán, 2006; BCG y WFPMA, 2008, 2010, 2012; López Núñez, 2008; Sánchez Augier, 2009; Soltura Laseria, 2009; Hernández Junco, 2009; Nieves Julbe, 2010; Parra Villanueva, 2010; Stable Rodríguez, 2012; Monagas Docasal, 2012; Montejo Salazar, 2013; Cuesta Santos, 2014. 
organización del trabajo, evaluación del desempeño, capacitación y desarrollo, así como seguridad y salud del trabajo. Además comenzaba la implantación de las normas ISO 18000. Por el trabajo realizado en la actividad, el hotel fue la primera entidad de Cubanacán que certificó el uso de los sistemas de: Gestión de Seguridad y Salud del Trabajo, Gestión de Calidad y Gestión Medioambiental.

\section{Etapa I. Preparación}

Se enfatizó a través de los tres pasos de la etapa en la tenencia del equipo encargado del trabajo a desarrollar en la EDI, su preparación, conocimiento y forma de ejecutar dicho tema en la entidad.

Paso 1. Crear el comité de competencias para el sistema de evaluación del desempeño individual

El comité se conformó con el director del área de Capital Humano y el Especialista Principalde la misma así como 1 representante de la dirección de cada proceso que se desarrolla en la organización (comercialización, servicios gastronómicos, animación, alojamiento, servicios técnicos, logística y economía). Además se incluyeron dos trabajadores de experiencia del área y un representante del sindicato.

Paso 2. Capacitar el comité de competencias para el sistema de evaluación del desempeño individual

Antes de iniciar los trabajos de diseño el comité se familiarizó con las tendencias más actuales de la GCH: la gestión por competencias; los perfiles de cargos, como sostén de la planeación cualitativa a este nivel; la necesidad del carácter sistémico en la organización, en general y de la $\mathrm{GCH}$, en particular (comprobaron que estuvieran identificados los subsistemas, la relación entre ellos y su grado de contribución al objetivo final de la misma). Además se explicó el término de conductas estratégicas dentro del concepto de competencias y en el perfil de cargo. Se dedicó varios espacios para visualizar al CMI como un sistema interactivo en la organización y el arte necesario para definir los indicadores de éste.

También se revisaron las resoluciones rectoras de este tema en el MTSS y el MINTUR. Una vez creada la necesaria base teórica se procedió a explicar cada una de las fases, etapas, pasos y tareas que integran el procedimiento.

Paso 3. Caracterizar la entidad turística, la evaluación del desempeño individual y analizar la influencia del entorno

El Hotel Brisas Guardalavaca, perteneciente al grupo de turismo Cubanacán, fue inaugurado el 26 de noviembre de 1994. Está situado en el polo turístico Guardalavaca a $54 \mathrm{Km}$ de la ciudad de Holguín. Ofrece servicio "Todo Incluido" con categoría cuatro estrellas. Está dirigido al turismo de sol y playa, con predominio al segmento de familia. El hotel cuenta con 231 habitaciones estándar (con vistas al jardín y mar) y una villa con nueve bloques. Ésta última posee otras 126 habitaciones estándar y 80 mini suites. Sus 
decoraciones constituyen un alto atractivo al turista por la belleza e historia que representan (las nueve villas fundadas por Cristóbal Colón).

La comercialización se realiza de forma coordinada entre la Dirección Comercial de Hoteles Cubanacán y el hotel. La gestión de ventas de este último se realiza, fundamentalmente, a través de las agencias de viajes (AA.VV), ya sean mayoristas, minoristas o de ambas clasificaciones. Los principales mercados emisores son Canadá, Inglaterra y Alemania. Además de estos mercados se reciben turistas de otros países, aunque en menor cantidad, con tendencia al crecimiento. Entre ellos se encuentran Italia, Holanda, Suiza y Francia. El turismo nacional comienza a tener cierto incremento. La ocupación de la planta hotelera disponible es una de las mejores por año en el territorio, al 105\% como promedio en este periodo (últimos tres años) lo que la hace distintiva. Sus resultados económicos también lo confirman al aumentar en los últimos 5 años,como promedio, un $6 \%$ sus ingresos y un $4 \%$ las utilidades.

\section{Etapa II. Contextualización estratégica}

Esta etapa priorizó la orientación estratégica de la entidad, garantizando la armonía entre las áreas a partir de los objetivos derivados de su misión fundamental, mostrando la conectividad en cada uno de los niveles (entidad, procesos, cargos). Los cuatro pasos de esta etapa evidenciaron sus resultados.

Paso 4. Definir o redefinir la estrategia organizacional

Se constató la existencia de la misión, visión y objetivos estratégicos de la entidad, mediante entrevistas con representantes de la dirección y revisión de documentos existentes. El objeto social de la entidad aprobado por resolución superior fue un aspecto a tener en cuenta en estas definiciones.

Misión: "Hotel Brisas Guardalavaca, el todo incluido más cubano. Ofrece un servicio de calidad con precios razonables, que satisface sus expectativas en un ambiente familiar y natural. El equipo de trabajo profesional y hospitalario le espera para compartir juntos una experiencia inolvidable, que lo hará retornar en sus próximas vacaciones".

Visión: "Hotel Brisas Guardalavaca, el todo incluido más popular del Caribe, caracterizándonos por la hospitalidad y diversión sin límites".

Objetivos estratégicos hasta el 2018:

1. Lograr un crecimiento sostenido en la eficiencia económica para ser el hotel de mayor utilidad de la marca Brisas.

2. Mantener y mejorar las capacidades instaladas asegurando el desarrollo de un producto sostenible y de alta competitividad.

3. Incrementar los niveles de ocupación y encauzar la política comercial a mercados y sectores de mayor poder adquisitivo.

4. Lograr que los trabajadores asuman los valores compartidos, donde se destaque el sentido de compromiso como parte de la cultura empresarial y preservar la moral revolucionaria de los cuadros y trabajadores. 
5. Potenciar la gestión del capital humano como vía para asegurar el desarrollo y la preparación de los trabajadores bajo el concepto de organización que aprende, donde se logre trabajadores motivados y profesionales.

6. Desarrollar una cultura integral de la calidad con la optimización de procesos clave de trabajo como comercialización, restauración y logística, que asegure el crecimiento sostenido de la satisfacción y fidelidad de los clientes.

7. Alcanzar la excelencia del servicio con el $100 \%$ del personal idóneo según perfiles de competencias de los cargos que ocupan.

Paso 5. Diseño o rediseño de los procesos de trabajo turísticos

La entidad aplicaba el enfoque por procesos como se puede observar en su Mapa. En éste se especifican las principales entradas, transformaciones y salidas, los sistemas de control y formulación de priorización al logro de los resultados finales de la entidad. Se constató que todos se encontraban en función de la misión, visión y objetivos estratégicos, antes explicados.

Paso 6. Diseño del control organizacional estratégico

Para la búsqueda permanente de las oportunidades de mejora del sistema y cómo asegurarla, se aplicó como instrumento de control para medir los resultados de forma general y específica a todos los niveles, el CMI. El mismo permitió complementar los indicadores de medición de los resultados de actuación de la organización con indicadores financieros y no financieros de los factores claves que influirán en los resultados del futuro de cada puesto, área y proceso.

Se partió del mapa de la organización que responde a los objetivos estratégicos generales. Este CMI causal (también se le conoce así) permitió hacer la derivación de los indicadores generales que el hotel debía evaluar a nivel organizacional, quedando expresado a través del CMI a nivel estratégico o referencial. Además se operativizó el cuadro de mando integral a nivel táctico (de los procesos.

Paso 7. Diseño o rediseño de los perfiles de cargos por competencias

Por ser el diseño del perfil del cargo por competencias decisivo en esta conducción metodológica, se tuvo en cuenta como parte del proceso de evaluación del desempeño. Se revisó mediante documentos si estaban definidos, así como el grado de calidad que presentaban. Aunque estos se encontraban diseñados, no tenían orientación de las competencias hacia los indicadores de conductas estratégicas (se utilizó la técnica participativa Delphi por rondas para establecer las mismas) que permitirían conectarlos a los objetivos estratégicos de la organización. Además, serían la base del conjunto de procesos claves en la GCH.

\section{Fase II. Transformación.}

Esta fase posibilitó profundizar en el diseño de la EDI y su aplicación, unido a la obtención de los resultados del desempeño organizacional estratégico a través del CMI operativizado en sus diferentes niveles. 


\section{Etapa III. Diseño del sistema de evaluación integrada del desempeño individual}

Esta etapa permitió, con sus cuatro pasos, obtener información oportuna y adecuada para la toma de decisiones posteriores en los diversos procesos que se ejecutaron en la entidad. Por lo tanto estableció la conexión sistémica necesaria.

Paso 8. Determinar los indicadores y las conductas estratégicas

Se establecieron los indicadores que muestran los resultados a lograr por el trabajador. Lo que significa una expresión del grado en que se ofreció respuesta a la derivación de los objetivos de la organización con su misión o razón de ser del cargo, a partir de sus conductas estratégicas. Estas últimas quedaron establecidas en este paso.

Paso 9. Diseñar el mecanismo para recopilar la información

Según la naturaleza de los indicadores propuestos, las características de la actividad, los intereses de la organización y el sistema de registros de la información, se propone la frecuencia, fuente y registro

Paso 10. Establecer la correlación entre indicadores

Para desarrollar este paso se valoró el cargo de dependiente partiendo de la clasificación de los procesos, su mapa y el lugar de éste en la organización. La información obtenida en las tablas de doble entrada se logró con la aplicación del método de consenso con el uso de la Moda. Primero, se estableció la correlación con los indicadores de procesos teniendo en cuenta la influencia (alta media, baja) de los indicadores del cargo sobre los indicadores de los procesos.

Paso 11. Determinar la importancia relativa del indicador

A través de la utilización de método de experto de Kendall se logra establecer la importancia relativa de los indicadores.

\section{Etapa IV Desempeño del sistema de evaluación}

En esta etapa se procedió a poner en práctica todo lo diseñado en la anterior. Se desarrolló la evaluación del desempeño registrándose toda la información que posteriormente sería la base de múltiples análisis, decisores de medidas importantes para la mejora en la actuación individual y organizacional.

Paso 12. Recopilar la información

Se realizó la recopilación de la información necesaria para caracterizar el desempeño de los trabajadores, los procesos, la organización de acuerdo con los instrumentos generados en el paso 9, según método, frecuencia y fuentes diseñadas.

Paso 13. Evaluar el desempeño organizacional estratégico

Se realizó la evaluación de este desempeño, desde el CMI operativizado en los niveles referencial y táctico; valorados a través de sus indicadores, el estado deseado y el 
alcanzado. Para lograr una calificación final de cada uno de ellos, se ejecutó la tarea de homologarlos, lo que resultó llevarlos a una misma escala.

Paso 14. Evaluar integradamente a los trabajadores

El comité ejecutó las dos tareas fundamentales diseñadas para este paso. Para ello utilizó la calificación de las conductas estratégicas del cargo en superior, aceptable o deficiente y la información ofrecida de los pasos anteriores. El ajuste e integración de la evaluación se realizó como se explica a continuación.

\section{Tarea 3. Ajustar los indicadores}

Con la homologación y evaluación de los indicadores de los procesos, de la organización, así como el grado de correlación entre éstos y los indicadores de conductas estratégicas establecidas en el cargo, se procedió a ajustar la evaluación de estos últimos.

\section{Tarea 4. Integrar los indicadores}

Según la evaluación ajustada de los indicadores del cargo otorgada en el paso anterior, así como la Importancia relativa dada a cada uno de ellos, se obtiene la evaluación integrada del trabajador.

Resulta interesante ver de forma integrada la evaluación alcanzada en: el área de este dependiente Restauración 1), los demás que conforman este cargo, así como el proceso a que éstos pertenecen, otros que influyen en este desempeño; así como la organización. Así se puede valorar las relaciones entre resultados y causas, expresado a través de estos indicadores.

\section{Fase III. Salida}

En la misma se logró la integración interna y externa de la EDI, a los procesos de la GCH y hacia los demás procesos del hotel, profundizando en las causas del resultado de la evaluación y la interconexión multicausal de los mismos hacia los resultados generales esperados. A través de las dos etapas de esta fase, se reafirmaron los aspectos inhibidores del desempeño individual y organizacional. Se destacaron los factores claves del éxito, además de realizar un plan de mejora para la organización y para el propio proceso de.EDI.

\section{Etapa V. Explotación de los resultados de la evaluación}

Resultó significativa la aplicación de esta etapa pues se logró realizar un análisis integrado de las causas que afectaron la evaluación del trabajador en su cargo. Además de valorar los nexos existentes entre esta EDI y demás conexiones técnico organizativas del sistema de GCH.

Paso 16. Analizar los factores inhibidores del desempeño

Se analizaron los factores inhibidores desde la mirada del capital humano, dividiendo la información en las condiciones técnico organizativas, así como en el estado motivacional y del saber. 


\section{Tarea 6. Analizar el estado motivacional y del saber}

Se les aplicó a los trabajadores del área la encuesta de satisfacción laboral pudiendo comprobar las insatisfacciones con las condiciones técnico organizativas y de aseguramiento, por insuficiencias con los medios de trabajo y los insumos para dar un servicio de excelencia. También consideraron la existencia de barreras en la superación profesional por las características del régimen de trabajo. Todos expresaron alta insatisfacción con la estimulación moral y material. Se reconoció el poco significado del salario y del sistema de pago respecto al trabajo realizado.

Paso 17. Realizar el programa de retroalimentación

Teniendo en cuenta los elementos antes expuestos se realizó un programa de acciones que incluyó la información de la evaluación final de los trabajadores, la firma de las mismas en el modelo establecido, así como los elementos que se debían mejorar en cada puesto de trabajo, procesos y organización. De esta forma se logró la existencia de una retroalimentación enfocada hacia la mejora continua, donde se valoró también los restantes procesos de la GCH y cómo podía vincularse los resultados hasta aquí abordados con cada conexión técnico organizativa de la misma.

\section{Etapa VI. Mejora del proceso de evaluación del desempeño individual}

Para profundizar en la calidad del proceso desarrollado se aplicaron los tres pasos considerados en esta etapa. Se midió el impacto del proceso en términos de cantidad de cargos evaluados, mejora producida en el desempeño de los cargos y satisfacción de los trabajadores con la evaluación además de la coherencia en la evaluación. Además se evaluó el vínculo del desempeño estimado y los resultados de la organización. Por último se ejecutó un programa de mejora que permitió actuar sobre las brechas existentes para elevar el desempeño y su calidad.

Se compararon los resultados obtenidos en la primera aplicación del procedimiento y en cada año posterior a su utilización.

\section{Conclusiones.}

Del análisis realizado en el estudio se puede concluir que:

La introducción de una concepción alineada en la EDI, que reconozca la interacción entre los cargos, los procesos y la organización, sobre la base de las conductas estratégicas en las competencias del cargo, otorga mayor coherencia a los resultados de la evaluación del desempeño.

> El uso del CMI operativizado en sus tres niveles (estratégico, táctico y operativo), con el despliegue de indicadores intangibles del capital humano, profundiza en las causas que limitan el desempeño y ofrece mayor pertinencia a la información que resulta de este proceso evaluativo. 
La aplicación integral del procedimiento propuesto en las organizaciones objeto de estudio, evidenció su factibilidad y conveniente utilización, como un instrumento metodológico para mejorar la gestión y su proceso actual de toma de decisiones.

\section{Referencias Bibliográficas.}

Cuesta Santos, A. Evaluación del Desempeño y compromiso en la gestión del capital humano. Instituto Superior Politécnico José Antonio Echeverría, La Habana, 2014, ISBN: 978-959-261-463-5

Browne, A (2014). Intervención de GastonAlphonsoBrowne, primer ministro de Antigua y Barbuda, presidente en ejercicio de CARICOM, en la clausura de la V Cumbre CARICOM-Cuba, La Habana, 8 de diciembre del 2014

Sánchez Augier, L. y otros (2009). Tecnología para la evaluación del desempeño de los recursos humanos. Aplicación en entidades hoteleras del destino Holguín. Monografía Universidad de Holguín

Delgado Pérez, E. (2003). Metodología para la realización del diagnóstico de la Gestión de los Recursos Humanos en empresas en Perfeccionamiento Empresarial. Holguín

Sotolongo Sánchez, M (2005). Procedimientos para la Auditoría Interna del Sistema de Gestión de Recursos Humanos en Instalaciones Turísticas Hoteleras Cubanas. Aplicación en Pequeñas y Medianas Instalaciones Turísticas Hoteleras. Tesis presentada en opción al Grado Científico de Doctor en Ciencias Técnicas. Universidad Central "Marta Abreu" de Las Villas. Cuba

Varela Izquierdo, N. (2001). Gestión Turística. Perfeccionamiento de los Recursos Humanos en el sector hotelero. Tesis en opción al grado científico de Doctor en Ciencias Técnicas. Instituto Superior Politécnico "José Antonio Echevarría" .Ciudad de la Habana, Cuba.

Nieves Julbe, A.F (2010).Procedimiento para Implantar el Ambiente de Control a través de Procesos Claves del Sistema de Gestión Integrada del Capital Humano. Tesis presentada en opción al grado científico de Doctor en Ciencias Técnicas, Universidad de Holguín “Oscar Lucero Moya”, Cuba

Stable Rodríguez, Y (2012). Modelo y metodología de aprendizaje organizacional para el mejor desempeño de una organización de ciencia e innovación tecnológica. Tesis presentada en opción al Grado Científico de Doctor en Ciencias Técnicas. Instituto superior de tecnologías y cienciasaplicadas. La Habana, Cuba

Comas Rodríguez, R (2013). Integración de herramientas de control de gestión para el alineamiento estratégico en el sistema empresarial cubano. Aplicación en empresas de Sancti Spíritus. Tesis presentada en opción al grado científico de Doctor en Ciencias Técnicas, Universidad de Matanzas "Camilo Cienfuegos". Cuba 


\section{Para citar el artículo indexado.}

Leyva C. (2019) Alineación de la evaluación del desempeño individual a la gestión hotelera. Revista electrónica Explorador Digital 3(3), 51-61. Recuperado desde: http://cienciadigital.org/revistacienciadigital2/index.php/exploradordigital/article/view/443/9 $\underline{99}$

\section{¿Ciencia}

El artículo que se publica es de exclusiva responsabilidad de los autores y no necesariamente reflejan el pensamiento de la Revista Explorador Digital.

El articulo queda en propiedad de la revista y, por tanto, su publicación parcial y/o total en otro medio tiene que ser autorizado por el director o editor de la Revista Explorador Digital.
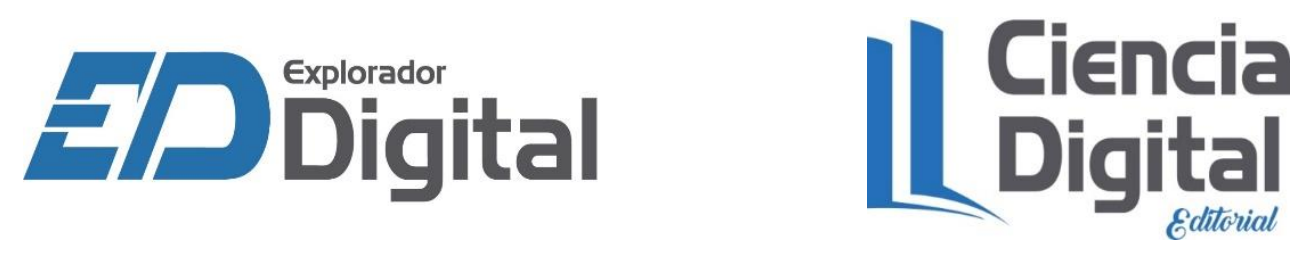